\title{
Avaliação da qualidade de vida em idosos pós- fratura da extremidade proximal do fêmur
}

\section{Evaluation of quality of life in the elderly with proximal femural fracture}

Amauri Milton Machadoํ, Alfésio Luís Ferreira Braga², Maria Lúcia Bueno Garcia ${ }^{3}$, Lourdes Conceição Martins ${ }^{1,2}$

\section{RESUMO}

Introdução: A fratura proximal do fêmur é causa de alta morbimortalidade tardia, comprometendo a funcionalidade e podendo afetar a qualidade de vida, principalmente em idosos. Objetivo: Avaliar a qualidade de vida de idosos após alta hospitalar por cirurgia de fratura proximal do colo do fêmur da região do Grande ABC paulista. Método: Estudo transversal com pacientes seis meses pós-cirurgia de fratura da extremidade proximal do fêmur realizada em dois hospitais pertencentes ao sistema de saúde suplementar no município de Santo André (SP) no período de 2006 a 2009, por meio de dois questionários: um sociobiodemográfico e o de qualidade de vida SF-36. Os dados foram submetidos à análise descritiva e teste $U$ de Mann-Whitney. 0 nivel de significância foi 5\%. Resultados: Foram entrevistados 50 pacientes entre 60 e 97 anos, média de 74,5 (desvio padrão=10,8). Houve predominância do sexo feminino (68\%), viúvas (52\%), na cor branca (70\%) e com nível de instrução até o Ensino Fundamental incompleto (62\%). As doenças mais prevalentes foram a hipertensão arterial sistêmica e osteoartrite. Com relação à qualidade de vida, houve diferença entre os sexos. Os homens apresentaram melhor escore para os domínios capacidade funcional $(p<0,001)$, dor $(p=0,025)$, aspectos físicos $(p=0,023)$ e aspectos sociais $(p=0,050)$, enquanto as mulheres pontuaram melhor em saúde mental $(p=0,024)$. Conclusão: Entre os idosos da região do Grande ABC paulista, após longo período do procedimento cirúrgico para fratura de colo de fêmur, observou-se melhor qualidade de vida para pacientes do sexo masculino quando comparados ao feminino.

Palavras-chave: qualidade de vida; idoso; fraturas do fêmur.

\begin{abstract}
Introduction: The proximal femur fracture is responsible for late high morbid-mortality rate, which may affect the quality of life, mainly in the elderly. Objective: To evaluate the quality of life of elderly patients from the Grande ABC paulista in the late post operatory period of surgery of fracture of the proximal end of the femur. Method: It is a cross-sectional study, conducted on patients six months after surgery of fracture of the proximal end of the femur held in two hospitals that belong to the additional health system in the municipality of Santo André (SP, Brazil) during 2006-2009. We applied two questionnaires: the SF-36, about quality of life, and a biodemographic survey. Data were analyzed by descriptive and Mann-Whitney $U$ tests. The significance level was $5 \%$. Results: A total of 50 patients were recruited, aged between 60 and 97 years, average 74.5 (standard deviation=10.8). There was a female predominance (68\%), widowed (52\%), white (70\%) and last than eight years of education (62\%). The most prevalent diseases were systemic hypertension and osteoarthritis. Men presented better score than women for functional capacity $(p<0.01)$, pain $(p=0.025)$, physical aspects $(p=0.023)$ and social aspects $(p=0.050)$ domains, while women scored better on mental health $(p=0.024)$. Conclusion: Among older people, after long period of surgical procedure for fracture of neck of femur, it was found a better quality of life for male patients compared to females.
\end{abstract}

Keywords: quality of life; aged; femoral fractures. 


\section{INTRODUCุÃO}

O aumento da longevidade mundial obriga as entidades médicas e sociais a se prepararem em relação à profilaxia e aos cuidados com as consequências da senescência e da senilidade, como a instabilidade do equilíbrio, quedas e a incapacidade funcional ${ }^{1-6}$. As quedas, mesmo da própria altura, associadas a outros fatores de risco, entre eles a osteoporose e a longevidade, podem levar a fraturas, incluindo a do colo do fêmur, que apresenta alta prevalência e morbimortalidade ${ }^{7-12}$. Além disso, como o osso tem capacidade de transmitir a carga durante o movimento, a imobilidade induzida pós-fratura pode potencializar perda da integridade estrutural óssea, levando à deterioração funcional do membro acometido, e, como num ciclo vicioso, intensificar a incapacidade física ${ }^{10,13,14}$.

Estima-se que nos Estados Unidos são registradas mais de 350 mil fraturas de fêmur anualmente, o que representa custo aproximado de US\$ 6 bilhões, com a mortalidade entre idosos variando de 12 a $37 \%{ }^{15-17}$, índice semelhante aos registrados em outros locais do mundo ${ }^{18-21}$. Com o envelhecimento da população, esses números tendem a crescer, podendo dobrar até o ano de $2040^{15}$. No Brasil, a mortalidade apresenta estatística semelhante, oscilando entre 7 e $34 \%$, com média de $22 \%$, dependendo da região analisada ${ }^{3,9,22-29}$. Os custos nacionais por essa afecção, apesar de subestimados, também atingem altos valores, onerando o orçamento do sistema público de saúde, já tão limitado ${ }^{29,30}$.

De acordo com a literatura, dentre as doenças mais prevalentes entre os idosos encontra-se a fratura do fêmur ${ }^{11,18,19,31-35}$. A população predominante afetada é a feminina, com acometimento mais tardio que os homens, por apresentar maior longevidade, osteoporose e longo período de climatério ${ }^{18,19,25,35}$. Destaca-se também que as repercussões sobre o estado físico, mental e prognóstico são diretamente relacionadas com o tipo de fratura ${ }^{11,20}$, tempo de internação ${ }^{36}$, medicações utilizadas ${ }^{24}$ e suas condições clínicas prévias, como estado de nutrição ${ }^{11}$, funcionalidade ${ }^{27} \mathrm{e}$ comorbidades $^{3,12,13,24,37}$.

Os domínios da qualidade de vida (funcionalidade, independência, bem estar, alterações do humor) podem ser afetados com diferentes intensidades frente ao mesmo fator agressor, dependendo do gênero, e, como consequência, influenciar o tratamento, que deve ser individualizado ${ }^{38,39}$. Entretanto, estudos focando a qualidade de vida no idoso portador de fraturas de fêmur são raros na literatura, principalmente no território nacional ${ }^{38}$, mesmo quando nos referimos a grandes centros urbanos, incluindo a região da Grande São Paulo ${ }^{11,12,28}$. Portanto, o objetivo deste estudo é avaliar a qualidade de vida de pacientes idosos que realizaram cirurgia corretiva de fratura do fêmur na região do Grande ABC paulista, Brasil.

\section{MÉTODOS}

Foi realizado um estudo transversal para avaliar a qualidade de vida de idosos pós-cirurgia de fratura da extremidade proximal do fêmur utilizando-se dois questionários: o SF-36 (Short Form Health Survey 36) e um questionário sociobiodemográfico construído pelos pesquisadores. Todos os idosos que concordaram em participar do estudo assinaram o Termo de Consentimento Livre e Esclarecido. Este projeto foi aprovado pelo Comitê de Ética e Pesquisa da Faculdade de Medicina do ABC, sob n 014/2009.

Utilizou-se uma amostra não probabilística por conveniência, composta por pacientes operados em dois hospitais terciários pertencentes ao sistema de saúde suplementar (a saúde suplementar é composta pelos segmentos das autogestões, medicinas de grupo, seguradoras e cooperativas) no município de Santo André entre 2006 e 2009, submetidos à cirurgia corretiva de fratura da extremidade proximal do fêmur. A entrevista foi realizada seis meses após o procedimento cirúrgico. Para tanto, o pesquisador telefonava aos pacientes e agendava uma visita a fim que o participante pudesse responder aos questionários.

Foram excluídos os pacientes com comorbidades que poderiam interferir nos resultados (Alzheimer, infarto, AVC e tumores), os que não quiseram participar da avaliação, os politraumatizados, os que evoluíram para óbito (internados ou não) e os com fraturas patológicas por infiltração óssea e outras do fêmur que não acometeram o terço proximal.

O questionário de dados sociobiodemográficos continha informações sobre gênero, idade, cor, escolaridade, estado civil, tipo de moradia, renda, com quem mora, situação profissional, doenças concomitantes, hábito de tabagismo e acompanhamento médico regular (definido por consultas regulares eletivas com retorno agendado no período de três meses e atendimento pelo mesmo médico há pelo menos um ano).

O questionário de qualidade de vida SF-36 é amplamente utilizado para a avaliação geral da qualidade de vida ${ }^{40}$. Trata-se de um instrumento já traduzido e validado para o português ${ }^{40}$ composto de 36 perguntas que avaliam dimensões genéricas sobre a saúde nas últimas quatro semanas, o que representa valores humanos básicos relevantes na avaliação da qualidade de vida da população geral, como capacidade funcional (de realizar tarefas mais ou menos básicas como vestir-se, por exemplo), aspectos físicos (a extensão em que a saúde interfere em atividades diárias como o trabalho), dor, estado geral de saúde (EGS; forma como seria taxada a saúde), vitalidade (energia geral para desempenhar tarefas diárias), aspectos sociais (extensão em que a saúde interfere com atividades sociais), aspectos emocionais (extensão na qual os problemas emocionais interferem na vida diária) e saúde mental ${ }^{40}$.

\section{Análise estatística}

Foi realizada a análise descritiva de todas as variáveis do estudo. Das qualitativas houve apresentação de seus valores absolutos e relativos. E das quantitativas foram apresentados os valores de tendência central e de dispersão ${ }^{41}$.

Para se verificar a associação entre gênero e as variáveis qualitativas foi utilizado o teste do $\chi^{241}$. 
Para as variáveis quantitativas, a aderência à curva normal foi verificada por meio do teste de Kolmogorov-Smirnov, e a homogeneidade das variâncias pelo de Levene ${ }^{41}$.

Como as variáveis não apresentaram esses dois princípios satisfeitos, foi utilizado teste não paramétrico para a comparação entre os gêneros (teste U de Mann-Whitney), dos dados do questionário SF-36 e também na avaliação por idade ${ }^{41}$.

O nível de significância foi 5\%. E o pacote estatístico utilizado foi o SPSS 17.0 for Windows.

\section{RESULTADOS}

Foram convidados 319 pacientes, excluídos 269 (260 por comorbidades que poderiam interferir nos resultados e nove que não quiseram participar da avaliação) e recrutados para o estudo 50 indivíduos (15,67\%) que preenchiam os critérios de inclusão.

Os pacientes apresentaram média de $74,5(\mathrm{dp}=10,8)$ anos, variando entre 60 e 97, sendo que o grupo de mulheres registrou idade média maior do que o dos homens $(77,7 \pm 10,7$ e $67,8 \pm 7,9$ respectivamente, $\mathrm{p}=0,04)$.

As Tabelas 1 e 2 apresentam análises descritivas dos dados sociobiodemográficos. Na Tabela 1 pode-se verificar que houve preponderância de sexo feminino (68\%), casadas (46\%) ou viúvas (52\%), cor branca (70\%), com baixo nível de instrução - não alfabetizados ou com fundamental incompleto (62\%) e que não moram sozinhas (98\%) - a maior parte (47\%) vive com o cônjuge.

Tabela 1: Análise descritiva de dados biodemográficos

\begin{tabular}{|c|c|c|}
\hline & $\mathrm{n}$ & $\%$ \\
\hline \multicolumn{3}{|l|}{ Sexo } \\
\hline Masculino & 16 & 32,0 \\
\hline Feminino & 34 & 68,0 \\
\hline \multicolumn{3}{|l|}{ Estado civil } \\
\hline Casado & 23 & 46,0 \\
\hline Viúvo & 26 & 52,0 \\
\hline Divorciado & 1 & 2,0 \\
\hline \multicolumn{3}{|l|}{ Cor } \\
\hline Branca & 35 & 70,0 \\
\hline Negra & 5 & 10,0 \\
\hline Parda & 9 & 18,0 \\
\hline Indígena & 1 & 2,0 \\
\hline \multicolumn{3}{|l|}{ Escolaridade } \\
\hline Não alfabetizado & 15 & 30,0 \\
\hline Fundamental incompleto & 16 & 32,0 \\
\hline Fundamental completo & 3 & 6,0 \\
\hline Médio incompleto & 5 & 10,0 \\
\hline Médio completo & 5 & 10,0 \\
\hline Superior completo & 4 & 8,0 \\
\hline Pós-graduação & 2 & 4,0 \\
\hline \multicolumn{3}{|l|}{ Mora sozinho } \\
\hline Sim & 1 & 2,0 \\
\hline Não & 49 & 98,0 \\
\hline \multicolumn{3}{|l|}{ Com quem mora } \\
\hline Cônjuge & 23 & 46,9 \\
\hline Filho & 21 & 42,9 \\
\hline Outros & 5 & 10,2 \\
\hline
\end{tabular}

A Tabela 2 descreve a renda e hábitos e apresenta que a metade dos pacientes era responsável pelo próprio sustento e tinha renda familiar bruta acima de cinco salários mínimos. Quase a totalidade deles não tinha o hábito do fumo (96\%) e possuía convênio médico (92\%). A maioria era acompanhada em hospitais e tratada pelo mesmo médico (56\%).

Quanto aos principais desfechos de saúde, observou-se que 26 $(52,0 \%)$ dos pacientes tinham hipertensão arterial sistêmica, 14 $(28,0 \%)$ diabetes mellitus, 32 (64,0\%) osteoartrite, e 12 (24,0\%) outras doenças. Dos 50 entrevistados, 37 (74,0\%) afirmaram ir ao médico regularmente e 45 (90,0\%) utilizam medicação diária.

Pode-se observar que há diferença entre os gêneros para alguns domínios do questionário SF-36. Considerando a capacidade funcional $(p<0,001)$, aspectos físicos $(p=0,023)$, dor $(p=0,025)$ e aspectos sociais $(p=0,05)$, os homens apresentaram valores médios maiores do que as mulheres. Já no domínio saúde mental, as mulheres apresentaram média maior do que os homens $(\mathrm{p}=0,024)$.

Nos demais domínios, como estado geral de saúde, vitalidade e aspecto emocional, não foram observadas diferenças estatisticamente significativas entre os grupos (Tabela 3 ).

\section{DISCUSSÃO}

Este estudo demonstrou que idosos da região do Grande $\mathrm{ABC}$ paulista apresentam, no pós-operatório tardio de cirurgia corretiva de fratura proximal de fêmur, comprometimento de sua qualidade de vida, sendo isso mais intenso entre as mulheres.

Tabela 2: Análise descritiva sobre renda e hábitos

\begin{tabular}{|c|c|c|}
\hline & $\mathrm{n}$ & $\%$ \\
\hline \multicolumn{3}{|l|}{ Responsável pelo sustento } \\
\hline Próprio & 25 & 50 \\
\hline Cônjuge & 9 & 18 \\
\hline Filho & 16 & 32 \\
\hline \multicolumn{3}{|l|}{ Renda familiar bruta } \\
\hline Acima de 1 até 3 salários mínimos & 17 & 34 \\
\hline Acima de 3 até 5 salários mínimos & 12 & 24 \\
\hline Acima de 5 salários mínimos & 21 & 42 \\
\hline \multicolumn{3}{|l|}{ Rendimento } \\
\hline Sim & 44 & 88 \\
\hline Não & 6 & 12 \\
\hline \multicolumn{3}{|l|}{ Fumante } \\
\hline Sim & 2 & 4 \\
\hline Não & 48 & 96 \\
\hline \multicolumn{3}{|l|}{ Possui convênio } \\
\hline Sim & 46 & 92 \\
\hline Não & 4 & 8 \\
\hline \multicolumn{3}{|l|}{ Local de atendimento médico } \\
\hline Unidade Básica de Saúde & 6 & 12 \\
\hline Hospital & 42 & 84 \\
\hline Outro & 2 & 4 \\
\hline \multicolumn{3}{|l|}{ É acompanhado(a) pelo mesmo médico } \\
\hline Sim & 28 & 56 \\
\hline Não & 22 & 44 \\
\hline
\end{tabular}


Tabela 3: Análise descritiva para cada um dos domínios do SF-36 estratificada por gênero

\begin{tabular}{|c|c|c|c|}
\hline & Masculino & Feminino & Teste* \\
\hline \multicolumn{4}{|l|}{ Capacidade Funcional** } \\
\hline Média (desvio padrão) & $45,9(19,3)$ & $25,1(15,6)$ & \multirow{2}{*}{$p<0,001$} \\
\hline Mínimo-máximo & $0,0-75,0$ & $0,0-85,0$ & \\
\hline \multicolumn{4}{|l|}{ Aspectos físicos** } \\
\hline Média (desvio padrão) & $29,7(29,2)$ & $11,8(17,7)$ & \multirow{2}{*}{$p=0,023$} \\
\hline Mínimo-máximo & $0,0-100,0$ & $0,0-50,0$ & \\
\hline \multicolumn{4}{|l|}{ Dor** } \\
\hline Média (desvio padrão) & $58,6(26,3)$ & $41,4(25,6)$ & \multirow{2}{*}{$p=0,025$} \\
\hline Mínimo-máximo & $12,0-100,0$ & $0,0-100,0$ & \\
\hline \multicolumn{4}{|l|}{ Estado geral de saúde } \\
\hline Média (desvio padrão) & $56,9(20,9)$ & $60,3(20,7)$ & \multirow{2}{*}{$p=0,640$} \\
\hline Mínimo-máximo & $5,0-87,0$ & $15,0-92,0$ & \\
\hline \multicolumn{4}{|l|}{ Vitalidade } \\
\hline Média (desvio padrão) & $58,4(16,2)$ & $49,4(18,0)$ & \multirow{2}{*}{$p=0,290$} \\
\hline Mínimo-máximo & $35,0-95,0$ & $0,0-85,0$ & \\
\hline \multicolumn{4}{|l|}{ Aspectos sociais** } \\
\hline Média (desvio padrão) & $76,6(15,1)$ & $61,8(27,5)$ & \multirow{2}{*}{$p=0,050$} \\
\hline Mínimo-máximo & $37,5-100,0$ & $0,0-100,0$ & \\
\hline \multicolumn{4}{|l|}{ Aspecto emocional } \\
\hline Média (desvio padrão) & $31,3(30,1)$ & $34,3(42,2)$ & \multirow{2}{*}{$p=0,990$} \\
\hline Mínimo-máximo & $0,0-66,7$ & $0,0-100,0$ & \\
\hline \multicolumn{4}{|l|}{ Saúde mental** } \\
\hline Média (desvio padrão) & $53,0(23,3)$ & $66,8(17,4)$ & \multirow{2}{*}{$p=0,024$} \\
\hline Mínimo-máximo & $0,0-96,0$ & $20,0-88,0$ & \\
\hline
\end{tabular}

Elas apresentaram os piores resultados para os domínios funcionabilidade, independência física e sociabilização. Também registraram os piores escores funcionais físicos, que podem ser atribuídos, pelo menos em parte, à sua maior longevidade e acometimento tardio do evento ósseo. Os homens, por sua vez, tiveram prognóstico mais reservado no quesito saúde mental. Os resultados favorecem a proposta de mais atenção e cuidados à saúde mental de idosos masculinos após o tratamento da fratura. O questionário de qualidade de vida SF-36 se mostrou adequado aos nossos objetivos e nos indicou diferenças significativas entre homens e mulheres em diferentes domínios. Vários estudos aplicam o SF-36 com confiabilidade para a avaliação da qualidade de vida abrangente e geral em pacientes portadores de doenças, inclusive em procedimentos ortopédicos como a fratura de fê$\operatorname{mur}^{28,32,38}$. No Brasil, alguns autores optam por utilizar outros questionários de qualidade de vida e funcionabilidade mais específicos para avaliar o mesmo tipo de fratura ${ }^{3,11,27}$ ou associar a questionários mais específicos para artroplastias de quadril e joelho, como por exemplo o WOMAC e o Harris Hip Score ${ }^{32,39}$.

Os pacientes deste estudo pertencem à região do Grande $\mathrm{ABC}$ paulista, que faz parte da Grande São Paulo, maior cidade da América Latina e que apresenta a maior renda per capita, maior taxa de médico por habitante, alta longevidade e taxas de fraturas em idosos ${ }^{28}$. Esperávamos que os dados se comportassem semelhantemente às grandes metrópoles do mundo e diferentemente a de outros locais mais carentes do Brasil. Porém, na literatura há variação de dados de qualidade de vida, provavelmente pela heterogeneidade, assim como os de padrões de assistência, dificultando essa comparação $0^{31,34}$.
Optamos por analisar seis meses pós o ato cirúrgico na tentativa de afastar as consequências da inflamação aguda provocada pelo trauma da fratura e pelo ato cirúrgico, tentando verificar as consequências autoalimentadas das sequelas do processo crônico e dos eventos associados, como a dor, limitação funcional da senescência e possível senilidade, que podem acompanhar o envelhecimento. Vários autores nacionais ${ }^{3,9,11,12,27,39}$ e estrangei$\operatorname{ros}^{31,34}$ também optaram por averiguar o pós-operatório tardio, demonstrando a relevância da avaliação tardia deste processo. $\mathrm{Na}$ região do Grande $\mathrm{ABC}$ paulista encontramos apenas um estudo sobre qualidade de vida do pós-operatório tardio de colo de fêmur (um ano) em idosos ${ }^{28}$, porém suas análises se restringiram apenas a um tipo de artroplastia de quadril (artroplastia de Thompson) e não houve estratificação por gênero nas análises comparativas.

A maior incidência encontrada em nossos resultados foi de indivíduos brancos, aposentados, que moram com a família, o que está de acordo com a literatura ${ }^{11,13,15}$. Os quesitos "renda bruta maior que cinco salários mínimos” e "moradia própria” já eram esperados, pois o levantamento de dados foi realizado em hospitais de atendimento a pacientes conveniados e particulares, com status diferente de estudos com pacientes oriundos de instituições públicas ${ }^{11}$.

A predominância do sexo feminino está de acordo com a literatura, pois este gênero apresenta mais riscos de fratura de colo de fêmur, como longevidade, maior tempo de climatério e osteoporose $^{7,16-19,37}$. A longevidade e presença de comorbidades facilitaria a chance de quedas e fraturas, o que levariam ao aumento do sedentarismo, criando um ciclo vicioso de deteriorização funcional física e mental ${ }^{3,15,27,34}$. As doenças mais prevalentes encontradas na população estudada foram a hipertensão arterial sistêmica e a osteoartrite, provavelmente por serem essas as mais presentes em pessoas a partir de 65 anos $^{6,12}$. Acreditamos que nossos resultados subestimam as alterações na qualidade de vida pós-operatório tardio de fratura de fêmur, pois foram excluídos os pacientes com comorbidades com alterações do sensório, cognição, humor e déficits dos sentidos que poderiam afetar a estabilidade marcha e, consequentemente, interferir na qualidade de vida. A literatura mostra que essa população com mais comorbidades e limites para locomoção apresenta maior comprometimento funcional físico e mental na qualidade de vida pós-evento traumático, em especial procedimentos ortopédicos, internação e cirurgias, com sequelas maiores e piores prognósti$\cos ^{9,24,27}$. Entretanto, esta conduta permitiu a homogenização da população estudada permitindo maior chance de evidenciar as alterações que este estudo objetiva.

É possível que tenhamos encontrado prejuízo na qualidade de vida dos idosos quando verificamos os dados psicossociais, uma vez que a análise foi recordatória e, na maioria dos casos, os pacientes já se encontravam deambulando, porém com comprometimento de seu aspecto físico após seis meses da realização da cirurgia. Outros estudos evidenciaram o quarto mês após a fratura como o período de maior recuperação ${ }^{28}$. 
A queda da capacidade funcional e dos aspectos físicos, em que a maioria prefere realizar menos tarefas do que gostaria, com diminuição do tempo utilizado nestas atividades, ou não ter a capacidade de realizar as mesmas tarefas ou as que gostaria de realizar, pode ser um fator responsável pela queda de qualidade de vida encontrada em nossos pacientes. Entretanto, esses dados podem também refletir, em parte, que os pacientes idosos tendem a um excessivo apreço à sua condição de saúde para demonstrar autossuficiência e mais disposição como uma tentativa de não reconhecimento, ou não aceitação, ou não demonstrar às pessoas próximas as manifestações de seu declínio de estado geral.

Os pacientes não relataram interferência importante em suas relações familiares e sociais, o que pode ser explicado pela atenção que os idosos necessitam em função de seu quadro de pós-operatório de fratura do fêmur, exigindo mais cuidados físicos e psicossociais. Mas os mesmos referiram que sua saúde interfere de forma moderada no relacionamento familiar, apresentando-se como uma pessoa feliz, calma e animada durante a maior parte do tempo.

Interessantemente neste estudo houve alteração de qualidade de vida diferenciada com relação ao gênero. As mulheres apresentaram escores mais severos no quesito ligado à funcionalidade física e os homens, no domínio de saúde mental.

Acreditamos que a melhor funcionalidade física encontrada nos homens seja, em parte, devido à sua menor faixa etária quando ocorreu o evento da fratura, possibilitando melhor capacidade de reabilitação osteomuscular. Em concordância com esse raciocínio, a literatura aponta que a capacidade funcional decresce com o envelhecimento, aumentando as chances de queda, comorbidades e pior evolução de morbidades ${ }^{6}$. Porém, também neste quesito, os estudos são controversos. Alguns autores estão em concordância com nossos achados ${ }^{11}$ enquanto outros apresentam resultados diferentes, opostos ${ }^{31}$ ou semelhantes quando comparado ao gênero 9 .

Entre os homens, foi observada maior tolerância à dor e melhor score nos aspectos sociais. Interpretamos esses dados como consequências a fatores culturais, em que o gênero masculino usualmente apresenta mais repressão para expressar seus sentimentos e mais liberdade no aspecto social. Neste quesito, Rocha et al. ${ }^{9}$ pontuou que a dor é presente em $81 \%$ dos pacientes no primeiro mês pós-cirurgia, e apenas $10 \%$ persistem, necessitando de analgésicos após seis meses a um ano pós-cirurgia, porém não faz diferenciação em relação ao gênero 9 .
As mulheres, por sua vez, apresentaram melhores escores no domínio "saúde mental". O fato é muito interessante, pois sugere que apesar de maior idade, as mulheres conseguem manter qualidade no quesito psíquico, mesmo durante adversidades como eventos de internação, trauma ósseo e tratamento cruento como a cirurgia ortopédica. A literatura está em concordância com esses resultados, pois mostra que as mulheres apresentam maior longevidade e se mantêm mais ativas que os homens durante o envelhecimento com os afazeres de casa, com a família e mesmo no autocuidado, evitando o sedentarismo e postergando a disfunção psíquica-cognitiva ${ }^{13,27}$ e facilitando a manutenção de autonomia e qualidade de vida. A pior funcionalidade física encontrada neste grupo, e que sugere maior lentidão na reabilitação funcional depois do pós-operatório imediato da fratura de fêmur, poderia ser atribuída ao acometimento mais tardio do evento da fratura secundário à maior longevidade e melhor saúde mental.

Este estudo apresentou algumas limitações. Por ser transversal, reflete o que o grupo está sentindo no momento da coleta dos dados, não se podendo fazer inferências causais. Uma das maneiras encontradas para minimizar este problema foi a utilização de um questionário biodemográfico, no qual verificamos que o grupo de homens e mulheres apenas diferia com relação à idade. Outro fator limitante foi que todos os pacientes pertenciam a hospital terciário privado/conveniado, o que torna essa população com um perfil específico de classe econômica média-baixa, podendo interferir nas respostas do SF-36. Outro aspecto importante foi a perda de pacientes, pois por seguirmos alguns critérios rígidos já citados anteriormente, dos 319 levantados, obtivemos uma amostra final de apenas 50 pacientes. Além disso, houve grande predominância do sexo feminino, tornado a amostra absoluta de homens pequena e facilitando a ocorrência de erro tipo II.

Concluímos que entre os idosos da região do Grande $\mathrm{ABC}$ paulista, após longo período do procedimento cirúrgico para fratura de colo de fêmur, observou-se melhor qualidade de vida os do sexo masculino quando comparado ao feminino. Porém, para ambos ela se apresenta mais baixa do que para os idosos de mesma condição socioeconômica reportada na literatura. Os dados sugerem que o comportamento diferenciado dos idosos frente ao estresse citado pode ser uma ferramenta relevante para a tomada de decisões terapêuticas individualizadas, preconizadas para essa faixa etária. Consideramos também que os dados refletem o comportamento da população estudada.

\section{REFERÊNCIAS}

1. Ramos LR. Determinant factors for healthy aging among senior citizens in a large city: the Epidoso Project in Sao Paulo. Cad Saúde Pública. 2003:19(3):793-7.

2. Kirkwood RN, Gomes HA, Sampaio RF, Culham E, Costigan P. Análise biomecânica das articulações do quadril e joelho durante a marcha em participantes idosos. Acta Ortop Bras. 2007;15(5): 267-71.
3. Fortes S, Villano LAB, Lopes CS. Perfil nosológico e prevalência de transtornos mentais comuns em pacientes atendidos em unidades do Programa de Saúde da Família (PSF) em Petrópolis, Rio de Janeiro. Rev Bras Psiquiatr. 2008;30(1):32-7.

4. Barbosa MLJ, Nascimento EFA. Incidência de internações de idosos por motivo de queda em hospital geral em Taubaté. Rev Biociênc. 2001:7(1):35-42. 
5. Perracini MR, Ramos LR. Fatores associados a quedas em um corte de idosos residentes na comunidade. Rev Saúde Pública. 2002;36(6):709-16

6. Maciel ACC, Guerra RO. Prevalência e fatores associados ao déficit de equilíbrio em idosos. Rev Bras Ciênc Mov. 2005;13:37-44.

7. Cooper C, Barker DJ, Wickham C. Physical activity, muscle strength, and calcium intake in fracture of the proximal femur in Britain. BMJ. 1988;297(6661):1443-6

8. Sernbo I, Johnell O. Consequences of a hip fracture: a prospective study over 1 year. Osteoporos Int. 1993;3(3):148-53.

9. Rocha MA, Carvalho WS, Zanqueta C, Lemos SC. Estudo epidemiológico retrospectivo das fraturas do fêmur proximal tratados no Hospita Escola da Faculdade de Medicina do Triângulo Mineiro. Rev Bras Ortop. 2001:36(8):311-6.

10. Fabrício SCC, Rodrigues RAP, Costa Junior ML. Causas e consequências de quedas de idosos atendidos em hospital público. Rev Saúde Pública. 2004;38(1):93-9.

11. Garcia R, Leme MD, Garcez-Leme LE. Evolution of Brazilian elderly with hip fracture secondary to a fall. Clinics. 2006;61(6):539-44.

12. Hungria Neto JS, Dias CR, Almeida JDB. Características epidemiológicas e causas da fratura do terço proximal do fêmur em idosos. Rev Bras Ortop. 2011;46(6):660-7.

13. Muniz CF, Arnaut AC, Yoshida M, Trelha CS. Caracterização dos idosos com fratura de fêmur proximal atendidos em hospital escola público. Rev Espaço Saúde. 2007;8(2):33-8.

14. Vilas Boas Junior A, Soni J, Fratti SR, Kantovitz PCJ, Souza Filho RM, Valente Netto EB. A fratura do colo do fêmur como fator de maior morbidade e mortalidade. Rev Bras Ortop. 1998;33(6):483-8.

15. Souza RC, Pinheiro RS, Coeli CM, Camargo Jr. KR, Torres TZG. Aplicação de medidas de ajuste de risco para a mortalidade após fratura proximal de fêmur. Rev Saúde Pública 2007;41(4):625-31.

16. Ray NF, Chan JK, Thamer M, Melton LJ 3rd. Medical expenditures for the treatment of osteoporotic fractures in the United States in 1995: report from the National Osteoporosis Foundation.J Bone Miner Res. 1997:12(1):24-35

17. Burge R, Dawson-Hughes B, Solomon DH, Wong JB, King A, Tosteson A. Incidence and economic burden of osteoporosis-related fractures in the United States, 2005-2025. J Bone Miner Res. 2007;22(3): 465-75

18. Maravic M, Le Bihan C, Landais P, Fardellone P. Incidence and cost of osteoporotic fractures in France during 2001. A methodological approach by the national hospital database. Osteoporos Int. 2005;16(12):1475-80.

19. Morales-Torres J, Gutiérrez-Ureña S. The burden of osteoporosis in Latin America. Osteoporos Int. 2004;15(8):625-32.

20. Ioannidis G, Papaioannou A, Hopman WM, Akhtar-Danesh N, Anastassiades T, Pickard L, et al. Relation between fractures and mortality: results from the Canadian Multicentre Osteoporosis Study. CMAJ. 2009;181(5):265-71.

21. Dzupa V, Bartonícek J, Skála-Rosenbaum J, Príkazský V. Mortality in patients with proximal femoral fractures during the first year after the injury. Acta Chir Orthop Traumatol Cech. 2002;69(1):39-44.

22. Pereira GJC, Barreto AA, Curcelli EC, Pereira HR, Gérios JC, Galväo $\mathrm{MPL}$. Estudo epidemiológico retrospectivo das fraturas do terço proximal do fêmur na região de Botucatu. Rev Bras Ortop. 1993;28(7): 504-10.

23. Komatsu RS, Simões MF, Ramos LR, Szejnfeld VL. Incidência de fraturas do fêmur proximal em Marília, São Paulo, Brasil, 1994 e 1995. Rev Bras Reumatol. 1999;39(6):325-31.

24. Sakaki MH, Oliveira AR, Coelho FF, Leme LEG, Susuki I, Amatuzzi MM. Estudo da mortalidade na fratura do fêmur proximal em idosos. Acta Ortop Bras. 2004;12(4):242-9
25. Silveira VAL, Medeiros MMC, Coelho-Filho JM, Mota RS, Noleto JCS, Costa FS, et al. Incidência de fratura do quadril em área urbana do Nordeste brasileiro. Cad Saúde Pública. 2005:21(3):907-12

26. Pinheiro RS, Coeli CM, Vidal EIO, Camargo Jr KR. Mortalidade após fratura proximal do fêmur. Cad Saúde Colet. 2006;14(2):327-36.

27. Cunha U, Veado MAC. Fratura de extremidade proximal do fêmur em idosos: independência funcional e mortalidade em um ano. Rev Bras Ort. 2006;41(6):195-9.

28. Chikude T, Fujiki EN, Honda EK, Ono NK, Milani C. Avaliação da qualidade de vida dos pacientes idosos com fratura do colo do fêmur tratados cirurgicamente pela artroplastia parcial do quadril. Acta Ortop Bras. 2007:15(4):197-9.

29. Fernandes RA, Araújo DV, Takemoto MLS, Sauberman MV. Fraturas do fêmur proximal no idoso: estudo de custo da doença sob a perspectiva de um hospital público no Rio de Janeiro, Brasil. Physis. 2011;21(2):395-416.

30. Araújo DV, Oliveira JHA, Bracco OL. Custo da fratura osteoporótica de fêmur no sistema suplementar de saúde brasileiro. Arq Bras Endocrinol Metab. 2005;49(6):897-901.

31. Mesquita GV, Lima MALTA, Santos AMR, Alves ELM, Brito JNPO, Martins MCC. Morbimortalidade em idosos por fratura proximal do fêmur. Texto \& Contexto Enferm. 2009;18(1):67-73.

32. Soohoo NF, Vyas RM, Samimi DB, Molina R, Lieberman JR. Comparison of the responsiveness of the SF-36 and WOMAC in patients undergoing total hip arthroplasty. J Arthroplasty. 2007;22(8):1168-73.

33. SooHoo NF, Lieberman JR, Farng E, Park S, Jain S, Ko CY. Development of quality of care indicators for patients undergoing total hip or total knee replacement. BMJ Qual Saf. 2011;20(2):153-7.

34. Johnell O, Kanis JA. An estimate of the worldwide prevalence, mortality and disability associated with hip fracture. Osteoporos Int. 2004;15(11):897-902.

35. Samuelsson B, Hedström MI, Ponzer S, Söderqvist A, Samnegård E, Thorngren KG, et al. Gender differences and cognitive aspects on functional outcome after hip fracture - a 2 years' follow-up of 2,134 patients. Age Ageing. 2009;38(6):686-92.

36. Garbuz DS, Xu M, Duncan CP, Masri BA, Sobolev B. Delays worsen quality of life outcome of primary total hip arthroplasty. Clin Orthop Relat Res. 2006:447:79-84.

37. Formiga F, Navarro M, Duaso E, Chivite D, Ruiz D, Perez-Castejon $\mathrm{JM}$, et al. Factors associated with hip fracture-related falls among patients with a history of recurrent falling. Bone. 2008;43(5): 941-4.

38. Lopes AD, Ciconelli RM, Reis FB. Medidas de avaliação de qualidade vida e estados de saúde em ortopedia. Rev Bras Ortop. 2007;42(11/12):355-9.

39. Rampazo MK, D’Elboux MJ. A influência de variáveis sociodemográficas, clínicas e funcionais sobre a qualidade de vida de idosos com artroplastia total do quadril. Rev Bras Fisioter. 2010:14(3):244-51.

40. Ciconelli RM. Tradução para o português e validação do questionário genérico de qualidade de vida do Medical Outcomes Study 36-Item Short Form Healthy Survey (SF-36). Tese (Doutorado) - Universidade Federal de São Paulo, São Paulo, 1997.

41. Callegari-Jacques SM. Bioestatística: princípios e aplicações. Porto Alegre: Ed. Artmed; 2005.

\section{Endereço para correspondência}

Lourdes Conceição Martins - Rua Abraham Bloemaert, 126 - Jardim das Vertentes - CEP: 05541-320 - São Paulo (SP), Brasil - E-mail: lourdesc@usp.br.

Conflito de interesse: nada a declarar. 